\title{
The Effect of Dilation on Toric Intraocular Lens Aligning and Centring
}

\begin{abstract}
Purpose: To determine the effect of dilation on toric intraocular lens (IOL) alignment using conjunctival blood vessel or iris features and centration relative to the pupil.

Methods: Thirty-six patient's right eyes were imaged every two minutes after dilation with $0.5 \%$ Tropicamide. The orientation of conjunctival blood vessels on either side of the limbus and peripheral horizontal and vertical iris features were measured and compared to the magnitude of the dilation. The centration relative to the limbus and pupil shape were also assessed.

Results: The pupil was essentially symmetrical and remained so with dilation whereas the limbus was horizontally oval, remaining constant over time. Pupil dilation had no effect on the average orientation of the conjunctival blood vessels, horizontal or vertical iris features $(\mathrm{F}=2.95, \mathrm{p}=0.069)$, however, the variability between patients was much greater using iris features $(\mathrm{F}=31.233, \mathrm{p}<0.001)$. The change in pupil size was more strongly correlated to the change in orientation with the horizontal $(\mathrm{r}=$ $0.13 \pm 0.60)$ and vertical $(0.18 \pm 0.49)$ iris features than the conjunctival blood vessels $(\mathrm{r}=0.02 \pm 0.43 ; \mathrm{F}=3.149, \mathrm{p}=0.049)$. The pupil was on average centred superior $(0.07 \pm 0.09 \mathrm{~mm})$ nasal $(0.37 \pm 0.10 \mathrm{~mm})$ compared to the centre of the limbus, moving inferiorly with dilation.

Conclusion: Conjunctival blood vessel orientation is a more robust comparator for toric IOL surgical orientation monitoring than iris features as it is less reliant on pupil dilation. Pupil centration changes with dilation and should be considered when centring IOLs.
\end{abstract}

Keywords

Pupil Dilation; Toric Alignment; Toric Centration; Intraocular lens
Research Article

Volume 2 Issue 1 - 2015

\author{
Bhogal-Bhamra Gurpreet $\mathbf{K}^{1 *}$, \\ Wolffsohn James $\mathbf{S}^{2,3}$, Sheppard Amy $\mathbf{L}^{2,3}$, \\ Naroo Shehzad $A^{2,3}$, Shah Sunil ${ }^{2,4}$ and \\ Buckhurst Phillip ${ }^{5}$ \\ ${ }^{1}$ New Cross Hospital, UK \\ ${ }^{2}$ Ophthalmic Research Group, Aston University, UK \\ ${ }^{3}$ Aston Research Centre for Healthy Ageing, Aston \\ University, $U K$ \\ ${ }^{4}$ Midland Eye, UK \\ ${ }^{5}$ Plymouth University, UK \\ *Corresponding author: Gurpreet K Bhogal- \\ Bhamra, Wolverhampton Eye Infirmary, New Cross \\ Hospital, Wolverhampton, WV10 0QP, UK, Tel: 01902 \\ 69833; Email:preeti_bhogal@hotmail.co.uk
}

Received: December 01, 2014| Published: January 10 2015

\section{Abbreviations}

IOL: Intraocular Lens; Nd:YAG: Neodymium-Doped Yttrium Aluminium Garnet;CSO: Costruzione Strumenti Oftalmici

\section{Precis}

Conjunctival blood vessel orientation was shown to be a more robust comparator for toric intraocular surgical orientation monitoring than iris features as they are less reliant on pupil dilation.

\section{Introduction}

Astigmatism over 1.50 dioptres occurs in approximately $20 \%$ [1] and astigmatism greater than 2.00 dioptres occurs in approximately $8 \%$ of patients presenting for cataract surgery [2]. Although this astigmatism can be reduced by corneal or limbal relaxing incisions, toric intraocular lens (IOL) implants give a more predictable result and can correct higher levels of astigmatism [3,4]. It has been shown that uncorrected astigmatism can have a significant impact on the performance of daily tasks even if the mean spherical equivalent refraction is corrected, resulting in reduced quality of life [5]. Successful toric IOL implantation requires precise and accurate marking of the orientation at which the intraocular lens is to be implanted, as misalignment by as little as $1^{\circ}$ can result in an estimated $3.3 \%$ loss of cylindrical correction [6]. It is reported a deviation of $30^{\circ}$ with a toric IOL results in no correction of cylindrical power [7] and anything greater will add to the cylindrical power requiring correction [8].

The axis of astigmatic orientation or horizontal axis is generally marked on the peripheral cornea or over the sclera prior to surgery to account for the cyclorotation of the eye when moving to a supine position for the surgical procedure [9]. Ink markings often spread due to the blink action and tear film movement, and fade with time. Even superficial corneal lacerations used as markings have a finite size and rely on a surgeon's skill to be correctly located with reference to a bio microscope slit illumination orientation or protractor increments. Markings are usually made with patients sitting at a slit-lamp bio microscope, thus stable head positioning is critical in order to avoid misalignments due to head tilts [5]. There have been suggestions of using Nd:YAG laser to mark the cornea to overcome some of these issues [10], but head tilt again may prove a source of error. Imaging systems use conjunctival blood vessels or iris features as markers for orientation, but the robustness of this approach with variation in dilation during the imaging and surgery has not been investigated [11].

During refractive lens surgery and cataract extraction pharmacological dilation of the pupil is required to create an 
adequate opening for removal of the crystalline lens and insertion of the IOL. It is generally assumed that the pupil is circular, well centred within the iris and that dilation, caused by smooth muscle in the iris, occurs symmetrically. However, the pupil is generally located slightly nasal and superior to the geometric centre of the cornea and this changes with dilation, which is of clinical importance in ophthalmic surgery where pupil centration relative to the limbus can affect surgical outcomes $[12,13]$. The eye suffers a range of regular and irregular optical aberrations which can vary between eyes [12]. Both pupil dilation and decentration have been noted to increase high order aberrations within the visual system [13], in particular spherical aberration and coma [14], thus degrading retinal image quality. These aspects must be taken into consideration for various refractive procedures such as toric implantation as poor centration of an IOL will give rise to further optical aberrations resulting in poor image quality.

Various studies have been performed to investigate the changes in pupil centration with changes in diameter. Generally a superior-temporal shift in pupil centre has been found with pharmaceutically induced mydriasis and reports of inferiortemporal/temporal shifts with light induced mydriasis of magnitude $<0.2 \mathrm{~mm}$ [12-15]. Age does not appear to affect pupil centration [13]. There is, however, a well known linear decrease in pupil size with age which may account for the non-linearities in pupil shape that have been shown to increase with age [15].

Therefore this study has examined the effect of pharmaceutical dilation on conjunctival vessels and iris features, as potential markers for aligning toric IOLs. In addition, the changes in pupil centration relative to the limbus that occur with various degrees of dilation were assessed.

\section{Material and Methods}

Thirty-six subjects were recruited with a mean age of 21.67 \pm 2.73 years, 20 males and 16 females. All participants were healthy individuals with no ocular pathology. Exclusion criteria included no ocular abnormalities or pathology, no previous history of ocular surgery and no medication likely to affect rate or process of pupil dilation. Patients were required to maintain adequate posture as the procedure involved sitting still at a slit lamp for a duration of time.

After obtaining informed consent, intraocular pressures and Van-Herick angle depth measurements were performed prior to pharmaceutical dilation to ensure suitability of individuals for the investigation. Right eyes of each subject were then pharmacologically dilated using two drops of Tropicamide $0.5 \%$. Tropicamide $1.0 \%$ is more commonly used in surgery to ensure rapid maximal dilation, however the primary interest of this study was intermediate changes with dilation hence a weaker concentration was utilised. Ethical approval was granted by the Aston University Ethics Committee, the study also conformed to the tenets of the declaration of Helsinki.

Subjects were seated at a slit lamp where images of right eyes were taken every 2 minutes from immediately following instillation of the drops using an anterior eye imaging system attached to a C.S.O (Costruzione Strumenti Oftalmici, Scandicci, Italy) Elite slit lamp with high pixel light sensitivity. Images were captured for 30 minutes until maximal dilation was achieved [16]. Patients were instructed to keep as stable as possible during photographs.

The images were captured onto a computer linked to the imaging device, the camera exposure and image brightness were both controlled by Epsilon Lyrae software. Pairs of images were taken at each 2 minute time interval to allow for blurred images to be replaced. The pupils were illuminated by an external diffuse light source and a thin slit beam was positioned temporal to the limbus to minimise any influence on pupil diameter. At the end of the process intraocular pressure and angle measurements were repeated to ensure dilation had not induced angle closure glaucoma.

Analysis of the photographic images was then carried out using bespoke LabView ${ }^{\mathrm{TM}} 2010$ imaging software [11]. For each subject, pairs of the most prominent conjunctival vessels were chosen horizontally separated on either side of the limbus, and using LabView ${ }^{\mathrm{TM}}$ the orientation of these vessels were determined by drawing a line from one vessel to the other. Pairs of horizontal and vertical prominent features on the iris either side of the pupil were also selected for each participant, the orientation of which at each time point was determined by the same line drawing technique. Ovals were drawn to fit both the pupil and limbal margins to give the respective centration as well as width and height measurements in terms of $\mathrm{X}$ and $\mathrm{Y}$ coordinates. For pupils this was measured twice per image to give an average of height and width.

For repeatability purposes one randomly selected image per subject was re-analysed. As the values obtained were in pixel units a conversion factor determined from image analysing a millimetre unit ruler was applied ( 1 pixel $=0.01475 \mathrm{~mm}$ ) in order to convert the measurements to millimetres. Statistical analysis was then carried out using Analysis of Variance and Pearson's correlations as the data was normally distributed.

\section{Results}

The eye orientation and position between image capture every 2 minutes could vary. For example, at the typical slit lamp to eye distance of $30 \mathrm{~cm}$, a $0.2 \mathrm{~mm}$ change in limbal width (of $12.0 \mathrm{~mm}$ ) would occur from a $5 \mathrm{~mm}$ change in slit lamp to eye distance and a 0.02 change in limbal width to height ratio from a $11.4^{0}$ orientation change of the pupil to the camera plane. Therefore the average standard deviation of the 15 images for each subject captured every 2 minutes between dilation drug instillation and 30 minutes for the limbal width $(0.17 \pm 0.07 \mathrm{~mm})$ and height $(0.21 \pm 0.08 \mathrm{~mm})$ and the ratio between them $(0.02 \pm 0.01)$ were calculated. These variability values for the limbus were similar to the measurement variability of repeated analysis of the same image twice (limbal width $0.23 \mathrm{~mm}$; limbal height $0.22 \mathrm{~mm}$; ratio 0.03). Hence the eye orientation and position from the slit lamp camera seems to be well controlled by the conventional slit lamp head/chin rest and the slit-lamp focusing, and no compensation 
of the images captured between visits based on apparent limbal dimensions was therefore applied.

The limbus was generally vertically oval varying little with repeat imaging at each visit (width to height ratio $1.07 \pm 0.01$ ). The pupil was essentially symmetrical (width to height ratio $1.02 \pm 0.02$ at baseline), and remained so with dilation (SD \pm 0.013 ) as it increase in size in a sigmoid manner, with maximum dilation reached by approximately 25 minutes ( $y=-3.594 /(1+$ $\left.\mathrm{e}^{(-(-(x-13.18) / 3.569)}\right) r^{2}=0.998$; Figure 1). Standard deviations of repeat measurement of the pupil size were $0.12 \mathrm{~mm}$ for both width and height.

Pupil dilation had no significant effect on the average change

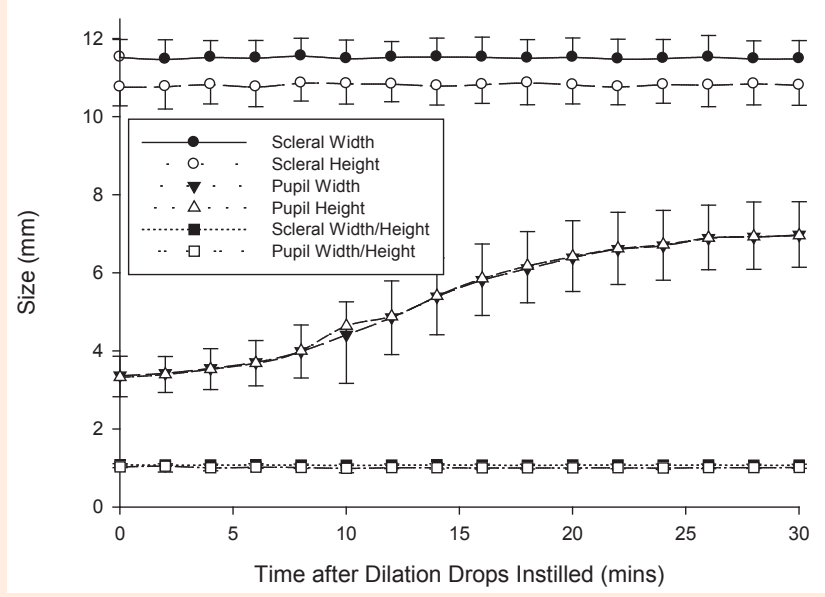

Figure 1: Variations in width and height of pupil, limbus and sclera with dilation. $\mathrm{N}=36$. Error bars $=1$ S.D.

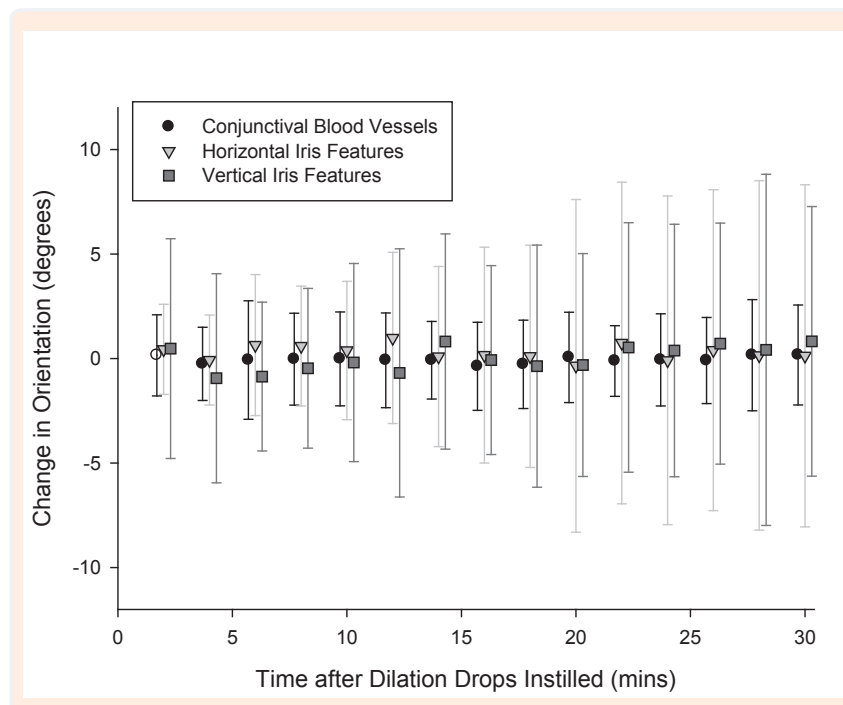

Figure 2: Orientation changes of conjunctival blood vessels and iris features through the process of dilation. $\mathrm{N}=36$. Error bars $=1$ S.D.

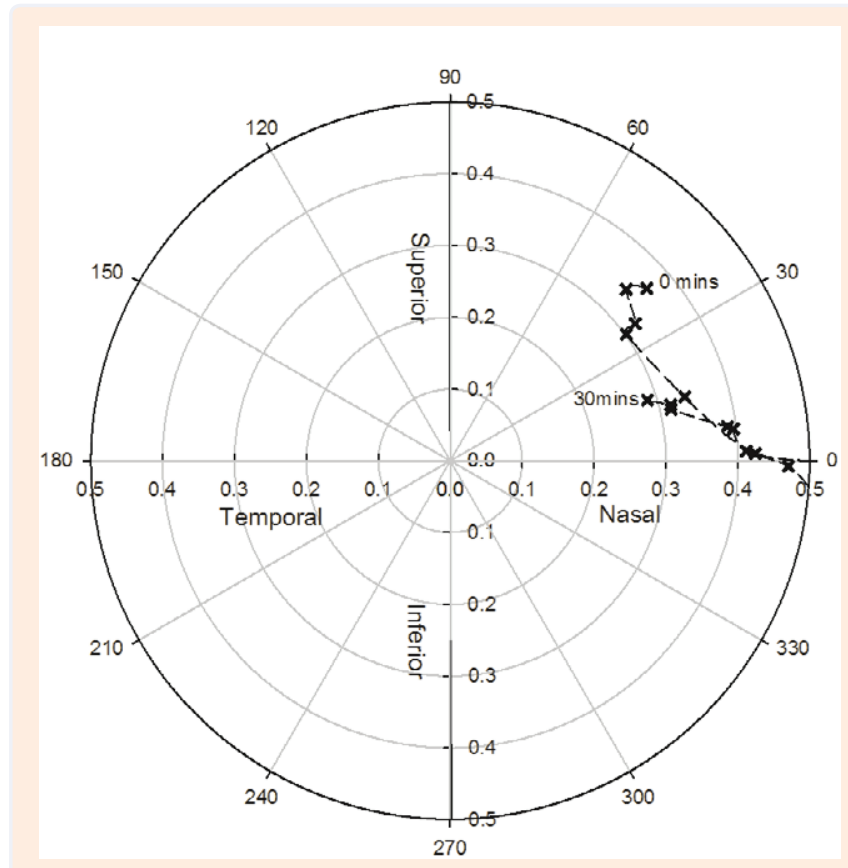

Figure 3: Changes in centration relative to the limbus ebvery 2 minutes with dilation. $\mathrm{N}=36$

in orientation of the conjunctival blood vessels, horizontal or vertical iris features $(F=2.95, p=0.069)$, however, the variability between subjects was much greater using iris features than conjunctival blood vessels $(F=31.233, p<0.001$; Figure 2). Standard deviations of repeat measurement of the orientation of the conjunctival blood vessels was $0.63^{\circ}$, and for the orientation of the iris features was $0.39^{0}$ horizontally and $0.87^{0}$ vertically. The change in pupil size was more strongly correlated to the change in orientation with the horizontal $(r=0.13 \pm 0.60)$ and vertical $(r=0.18 \pm 0.49)$ iris features than the conjunctival blood vessels $(r=0.02 \pm 0.43 ; F=3.149, p=0.049)$.

The pupil was on average centred superior $(0.07 \pm 0.09 \mathrm{~mm})$ nasal $(0.37 \pm 0.10 \mathrm{~mm})$ compared to the centre of the limbus, moving inferiorly with dilation with the change mainly occurring in the first 10 minutes post-dilation and some recovery from 20 minutes (Figure 3).

\section{Discussion}

The pupil, as expected, increased in diameter with pharmacological dilation reaching maximum dilation at approximately 25 minutes following drug instillation, the increase was generally symmetrical through the process with a sigmoidal increase in diameter. On analysis of pupil centration it was found to be located superior-nasal on average, moving inferior upon dilation, which coincides with previous studies of pupil centration [12-15]. It is possible that the changes in pupil centration may be smaller with physiological than pharmacological dilation [13], but the intraocular lens is implanted under pharmacological dilation so these pupil centration changes need to be considered. Previous research has shown these changes are not age dependant and 
hence the use of young subjects should not have influenced this conclusion. Non-linearity of the pupil was calculated as 0.018 which corresponds to that found by Wyatt [15] reporting 0.017 in dark conditions and 0.016 in light conditions.

Analysis of limbus height and width showed good repeatability over time, similar to that found on repeat analysis of the same images a second time. Therefore, this suggests there is adequate control of eye orientation using the head and chin rest of slit lamp with minimal effect from head tilt. The limbus remained generally oval in the horizontal plane which varied little upon repeat measurements. As surgeons generally use the pupil rather than the limbus to judge centration, such findings confirm the change in pupil centration when the eye is restored to physiological stimulation must be taken into consideration for surgery requiring dilation. If refractive surgery ablation extends beyond the pupil due to changes in centration with gelation, this can lead to glare, haloes and ghost images, hence resulting in an unsuccessful outcome [13] and dissatisfaction from the patient.

Previous research has shown image analysis of lens rotation to be more robust than subjective rating, and the use of conjunctival blood vessels or iris features to allow for head rotation further improves reliability [11]. Although dilation of the pupil had no effect on the average change in orientation of blood vessels and iris features, the variability of iris features with dilation was much greater in comparison to conjunctival blood vessels. The conjunctival blood vessels therefore prove to be better markers for orientation and hence provide surgeons with anatomical markers that can be used for various procedures.

Studies carried out to investigate the changes in pupil centration with diameter, generally report a superior-temporal shift in pupil centre with pharmaceutically induced mydriasis and inferior-temporal/temporal with light induced mydriasis of magnitude less than $0.2 \mathrm{~mm}$, which is only related to pupil size for the latter [12-15]. However, the effect of different degrees of dilation on pupil centration has not previously been assessed. This study showed a similar inferior centration shift finding with pharmaceutical dilation to that previously found with light induced mydriasis, although there was a counter movement superiorly from 20 minutes onwards. While the study was performed on younger adults to allow a wide dilation change to be imaged, age does not appear to affect pupil centration [13], although non-linearities in pupil shape increase with age [15]. Some surgeons use phenylephrine $2.5 \%$ in combination with $1.0 \%$ tropicamide and perhaps the use of a topical anaesthetic to speed dilation. Such additional pharmaceuticals may bleach and/ or dilate the conjunctival blood vessels, hence affecting their use for tracking head/eye rotation in toric IOL implantation.

In conclusion, it is well documented that shifts in pupil centration occur with pharmacological dilation which need to be considered for any type of refractive surgery to in order to avoid an unsuccessful outcome. The current study supports existing knowledge of this change in pupil location through dilation and has established conjunctival blood vessels as better markers for correcting the alignment of toric IOLs for head rotation than using iris features.

\section{References}

1. Ferrer-Blasco T, Montes-Mico R, Peixoto-de-Matos SC, GonzalezMeijome JM, Cervino A (2009) Prevalence of corneal astigmatism before cataract surgery. J Cataract Refract Surg 35(1): 70-75.

2. Hoffmann PC, Hutz WW (2010) Analysis of biometry and prevalence data for corneal astigmatism in 23,239 eyes. J Cataract Refract Surg 36(9): 1479-1485.

3. Sun XY, Vicary D, Montgomery P, Griffiths M (2000) Toric intraocular lenses for correcting astigmatism in 130 eyes. Ophthalmology 107(9): 1776-1781.

4. Mendicute J, Irigoyen C, Ruiz M, Illarramendi I, Ferrer-Blasco T, et al. (2009) Toric intraocular lens versus opposite clear corneal incisions to correct astigmatism in eyes having cataract surgery. J Cataract Refract Surg 35(3): 451-458.

5. Wolffsohn JS, Bhogal GK, Shah S (2011) Effect of Uncorrected Astigmatism on Vision. J Cataract Refract Surg 37(3): 454-460.

6. Lane S (2006) The AcrySof Toric IOL's FDA Trial Results. Cataract \& Refractive Surgery Today 5:66-68.

7. Shimizu K, Misawa A, Suzuki Y (1994) Toric IOLs: correcting astigmatism while controlling axis shift. J Cataract Refract Surg 20(5): 523-526.

8. Novis C (2000) Astigmatism and Toric Intraocular Lenses. Curr Opin Ophthalmol 11(1): 47-50.

9. Smith EM Jr, Talamo JH (1995) Cyclotorsion in the seated and supine patient. J Cataract Refract Surg 21(4): 402-403.

10. Bordonaba MC, Alvarez-Rementeria Fernandez L (2009) Marking the axis for toric IOL implantation. Cataract \& Refractive Surgery Today 8: 27-28.

11. Wolffsohn JS, Buckhurst PJ (2010) Objective Analysis of Toric Intraocular Lens Rotation and Centration. J Cataract Refract Surg 36(5): 778-782.

12. Walsh G (1988) The effect of mydriasis on the pupillary centration of the human eye. Ophthalmic Physiol Opt 8(2): 178-182.

13. Yang Y, Thompson K, Burns SA (2002) Pupil location under mesopic, photopic, pharmacologically dilated conditions. Invest Ophthalmol Vis Sci 43(7): 2508-2512.

14. Wilson MA, Campbell MCW, Simonet P (1992) The Julius F. Neumueller Award in Optics, 1989: Change of pupil centration with change of illumination and pupil size. Optom Vis Sci 69(2): 129-136.

15. Wyatt HJ (1995) The form of the human pupil. Vision Res 35(14): 2021-2036.

16. Krumholz DM, Portello JK, Rosenfield M, Rosenbaum JD (2006) A combination solution for routine pupillary dilation. Optometry 77(7): 350-353. 